\title{
Rapid detection and identification of mycobacteria from blood of patients with acquired immune deficiency syndrome
}

\author{
D. COLOMBritA, G. RAVIZZOLA, G. PINSI, R. LI VIGNI, F. PIRALI and A. TURANO
}

Institute of Microbiology, Brescia University, Piazza Spedali Civili 1, 25100 Brescia, Italy

Summary. A combination of radiometric broth culture (Bactec 13A) and the Genprobe nucleic acid hybridisation system was used to detect and identify Mycobacterium tuberculosis and $M$. avium from the blood of patients with acquired immune deficiency syndrome (AIDS).

\section{Introduction}

With the increased incidence of the acquired immune deficiency syndrome (AIDS), the isolation of mycobacteria from blood has become more frequent, ${ }^{1-3}$ but methods for their isolation and identification take several weeks. However, two products have become available recently that might provide a more rapid diagnosis-a radiometric broth (Bactec $13 \mathrm{~A}$; Johnston Laboratories Inc., Towson, MD, USA) specifically for the culture of mycobacteria from blood ${ }^{3}$ and a nucleic-acid hybridisation kit for the rapid identification of $M$. avium, $M$. intracellulare and the $M$. tuberculosis complex (Gen Probe Inc., San Diego, CA, USA). Identification is based on the ability of complementary nucleic acid strands to bond and form stable double-stranded complexes. ${ }^{4-6}$

The purpose of this study was to investigate the use of the combination of $13 \mathrm{~A}$ medium, recommended for the growth of mycobacteria from blood, ${ }^{3}$ and the Gen-probe system described as sensitive and specific for the identification of mycobacteria, ${ }^{6}$ to improve the diagnosis of mycobacterial infections in patients with AIDS and the AIDS-related complex (lymphadenopathy-associated syndrome and persistent generalised lymphadenopathy). ${ }^{7}$ Rapid detection and identification of $M$. avium and $M$. intracellulare is important because these micro-organisms are a major cause of disease in AIDS patients ${ }^{3}$ and because therapy for these patients differs from that used for patients infected with $M$. tuberculosis. ${ }^{6}$ Generally, most $M$. avium-intracellulare strains are resistant to isoniazid, rifampicin and quinolones but are susceptible to amikacin and gentamicin.

Received 21 Aug. 1989; revised version accepted 9 Feb. 1990.

\section{Materials and methods}

\section{Specimens}

From Jan. 1988 to May 1989, 875 blood specimens for the detection of mycobacteria were obtained from 153 patients with AIDS or associated syndromes, in Civili Hospital, Brescia.

\section{Culture methods and media}

Blood $(5 \mathrm{ml})$ was collected in a Bactec $13 \mathrm{~A}$ vial (Johnston Laboratories) and incubated at $36^{\circ} \mathrm{C}$. The vials were examined radiometrically daily on the Bactec 460 instrument; smears from samples with a growth index (GI) $>20$ were stained for acid-fast bacilli by the auramine method and examined by fluorescence microscopy. Positive samples were subcultured on LowensteinJensen and International Union (against) Tuberculosis Mycobacterium (I.U.T.M.) media (Sclavo S.p.a., Via Fiorentina 1, Siena, Italy).

\section{Identification}

Vials with a GI $>20$ and a positive auramine film were examined with the Gen-probe rapid diagnostic system. A 5-ml sample of a positive blood culture was centrifuged $(3200 \mathrm{rpm}$ ) for $10 \mathrm{~min}$ and $200 \mu \mathrm{l}$ of the pellet was added to a tube of lysing reagent and sonicated for $15 \mathrm{~min}$ at $50-70^{\circ} \mathrm{C}$ in a water-bath sonicator. Probe solution $(1 \mathrm{ml})$ was added and the tube was incubated for $1 \mathrm{~h}$ at $72^{\circ} \mathrm{C}$ in a water bath. Separation suspension $(4 \mathrm{ml})$ was added and incubated at $72^{\circ} \mathrm{C}$ for $5 \mathrm{~min}$ in a water bath; the tube was then centrifuged $(500 \mathrm{rpm})$ for $2 \mathrm{~min}$. Wash solution was added and the suspension was centrifuged ( $500 \mathrm{rpm}$ ) for $2 \mathrm{~min}$. The supernate was decanted and the tube was drained completely. Each tube was counted in a gamma counter for $1 \mathrm{~min}$ and hybridisation was calculated as follows : hybridisation $(\%)=($ sample $\mathrm{cpm}$ - background $\mathrm{cpm}) /($ total cpm of input probe - background $\mathrm{cpm}) \times$ 100 ; hybridisation $>10 \%$ was considered a positive 
result. The growth on Lowenstein-Jensen and I.U.T.M. media was identified by classical biochemical tests that included nitrate reduction, niacin and arylsulphatase production, catalase inactivation at $68^{\circ} \mathrm{C}$, photochromogenicity, tellurite reduction, Tween 80 hydrolysis and urease production.

\section{Antibacterial susceptibility tests}

In-vitro susceptibility tests for $M$. tuberculosis and $M$. avium were performed on $7 \mathrm{H} 11$ agar and isolates were considered susceptible when $\geqslant 99 \%$ reduction in the number of colonies was observed on the antimicrobial plate compared with the control plate. ${ }^{8}$

\section{Results}

Of the 875 blood cultures taken from 153 patients with AIDS or associated diseases $82(9.4 \%)$ cultures from 17 patients were positive for mycobacteria after an average time of $19 \cdot 2 \pm 7$ days. The mycobacterial isolates were identified by Genprobe-13 $(15.8 \%)$ from six patients as M. tuberculosis complex and $69(84.2 \%)$ from 11 patients as $M$. avium. The average growth time for $M$. tuberculosis was 27.4 SD7 days and for $M$. avium 17.8 SD5 days. Identification by the Gen-probe system took $c .2 \mathrm{~h}$. For $M$. tuberculosis, the mean percentage hybridisation was 28.45 SEM 2.06 with the homologous probe, 1.45 SEM 0.29 with the heterologous $M$. avium probe and 1.57 SEM 0.38 with the heterologous $M$. intracellulare probe. For $M$. avium isolates, the mean percentage hybridisation with the homologous probe was 33.72 SEM 5.92 , whereas with the heterologous $M$. tuberculosis complex and $M$. intracellulare probes the percentage hybridisation was 1.99 SEM 0.65 and 1.77 SEM 0.53 respectively. The percentage hybridisation with different probes for each of the 17 Mycobacterium strains is shown in the table. For comparison all the mycobacterial isolates grown on LowensteinJensen and I.U.T.M. media were identified by classical biochemical tests: 13 isolates that failed to grow on Lowenstein-Jensen medium within 7 days, produced niacin, reduced nitrate and produced catalase that did not withstand $68^{\circ} \mathrm{C}$ for $20 \mathrm{~min}$ were identified as $M$. tuberculosis $;{ }^{9}$ and 69 isolates that were slow growers at temperatures from 22 to $43^{\circ} \mathrm{C}$, produced smooth colonies that were not photochromogenic, became yellow on ageing, gave negative results for niacin production, nitrate reduction, Tween 80 hydrolysis, arylsulphatase activity, and urease production, and positive results for tellurite reduction and catalase activity at $68^{\circ} \mathrm{C}$ were identified as $M$. avium. ${ }^{10}$

Strains of $M$. tuberculosis were sensitive to etham- butol, streptomycin, rifampicin, isoniazid, ciprofloxacin and ofloxacin. $M$. avium strains were sensitive to ethambutol, streptomycin, amikacin and gentamicin.

\section{Discussion}

Of 17 cases of mycobacterial septicaemia in 153 patients with AIDS or associated syndromes, $15.8 \%$ were caused by $M$. tuberculosis and $84.2 \%$ by $M$. avium. Two of the cases had not shown any classical symptoms of septicaemia and were diagnosed unexpectedly by detection of atypical mycobacteria from blood emphasising the value of rapid detection. Although the time necessary for bacterial growth is not reduced in comparison with other systems such as Isolator ${ }^{3}$ the use of Bactec $13 \mathrm{~A}$ is advantageous in situations where it is desirable to avoid any further manipulations of the specimen, e.g., when taking blood at the bedside. Blood can be injected directly into the vial, ensuring greater accuracy of laboratory diagnosis and increased safety for the operators. The study also showed the reliability of the Gen-probe system, already known to be rapid and specific. ${ }^{5,6,11-13}$ The greatest criticism of the Gen-probe system is its high cost, but the advantage of being able to identify strains directly from a vial such as the Bactec 13A without waiting for growth of colonies on solid media is

Table. Nucleic acid probe hybridisation and classical biochemical identification of Mycobacterium strains

\begin{tabular}{|c|c|c|c|}
\hline \multirow[b]{2}{*}{$\begin{array}{c}\text { Strain } \\
\text { no. }\end{array}$} & \multicolumn{3}{|c|}{ Percentage hybridisation with probe for } \\
\hline & M. avium & $M$. intracellulare & $\begin{array}{l}\text { M. tuberculosis } \\
\text { complex }\end{array}$ \\
\hline \multicolumn{4}{|c|}{ M. tuberculosis } \\
\hline 1 & $1 \cdot 6$ & $1 \cdot 6$ & 27 \\
\hline 2 & $1 \cdot 3$ & $1 \cdot 3$ & 31 \\
\hline 3 & $1 \cdot 8$ & $2 \cdot 1$ & 31 \\
\hline 4 & $1 \cdot 7$ & $1 \cdot 9$ & $26 \cdot 3$ \\
\hline 5 & $1 \cdot 1$ & $1 \cdot 4$ & $28 \cdot 1$ \\
\hline 6 & $1 \cdot 2$ & $1 \cdot 1$ & $27 \cdot 3$ \\
\hline \multicolumn{4}{|c|}{ M. avium-intracellulare } \\
\hline 7 & 42 & $2 \cdot 1$ & $1 \cdot 8$ \\
\hline 8 & $20 \cdot 8$ & $1 \cdot 3$ & $1 \cdot 6$ \\
\hline 9 & $25 \cdot 7$ & $1 \cdot 5$ & $1 \cdot 3$ \\
\hline 10 & $37 \cdot 1$ & $2 \cdot 3$ & $1 \cdot 4$ \\
\hline 11 & $34 \cdot 2$ & $1 \cdot 2$ & 1.9 \\
\hline 12 & $35 \cdot 1$ & $1 \cdot 4$ & $2 \cdot 1$ \\
\hline 13 & $36 \cdot 1$ & $1 \cdot 6$ & $2 \cdot 2$ \\
\hline 14 & $34 \cdot 6$ & $1 \cdot 3$ & $2 \cdot 5$ \\
\hline 15 & $32 \cdot 3$ & $1 \cdot 8$ & $1 \cdot 1$ \\
\hline 16 & $33 \cdot 9$ & $2 \cdot 1$ & $3 \cdot 1$ \\
\hline 17 & $39 \cdot 1$ & $2 \cdot 9$ & $2 \cdot 9$ \\
\hline
\end{tabular}


considered to justify the expense. The use of the Gen-probe system increases the usefulness of Bactec 13A, emphasising its safety and practicality. Another criticism of the Gen-probe test is that identification of $M$. tuberculosis cannot be considered final because the probe identifies only the $M$. tuberculosis complex which includes $M$. africanum, $M$. bovis and $M$. microti. ${ }^{12}$ However, $M$. bovis is identified easily by negative results in niacin and nitrate tests; $M$. africanum is found only in tropical Africa; and $M$. microti occurs only in voles and other small mammals and may be regarded as a biovariety of $M$. tuberculosis. ${ }^{13}$ In our

\section{REFERENCES}

1. Macher AM, Kovacs JA, Gill V. Bacteremia due to Mycobacterium avium-intracellulare in the acquired immunodeficiency syndrome. Ann Intern Med 1983; 99 : $782-785$.

2. Salfinger M, Stool EW, Piot D, Heifets L. Comparison of three methods for recovery of Mycobacterium avium complex from blood specimens. J Clin Microbiol 1988; 26: 1225-1226.

3. Kiehn TE, Cammarata R. Comparative recoveries of Mycobacterium avium-M. intracellulare from Isolator lysis-centrifugation and BACTEC 13A blood culture systems. J Clin Microbiol 1988; 26: 760-761.

4. Drake TA, Hindler JA, Berlin OGW, Brukner DA. Rapid identification of Mycobacterium avium complex in culture using DNA probes. J Clin Microbiol 1987; 25: 1442--1445.

5. Gonzalez R, Hanna BA. Evaluation of Gen-probe DNA hybridization for the identification of Mycobacterium tuberculosis and Mycobacterium avium-intracellulare. Diagn Microbiol Infect Dis 1987; 8: 69-77.

6. Musial CE, Tice LT, Stokman L, Roberts GD. Identification of mycobacteria from culture using Gen-probe rapid diagnostic system for Mycobacterium avium complex and Mycobacterium tuberculosis complex. J Clin Microbiol 1988; 26 : 2120-2123. tests, all strains identified by the Gen-probe system as $M$ tuberculosis complex were confirmed as $M$. tuberculosis by classical biochemical tests.

AIDS patients infected with $M$. tuberculosis respond satisfactorily to standard antituberculous chemotherapy. This may not be so in infections due to $M$. avium as strains are often resistant to a wide variety of antituberculous drugs. It is useful, therefore, to be able to identify the infecting organism rapidly so that adjustments to chemotherapy may be made. What constitutes correct therapy for infections caused by $M$. avium remains to be decided.

7. Centers for Disease Control. Revision of the case definition of acquired immunodeficiency syndrome for national reporting United States. $M M W R$ 1985; 34: 373-375.

8. McClatchy JK. Antituberculosis drugs: mechanism of action, drug resistance, susceptibility testing and assays of activity in biological fluids. In: Lorian V. (ed) Antibiotics in laboratory medicine. Baltimore, Williams \& Wilkins Co. 1980: 135-169.

9. Kent PT, Kubica GP. Public health mycobacteriology: a guide for the level III laboratory. Atlanta, U.S. Department of Health and Human Services, Centers for Disease Control. 1985.

10. Wayne LG, Kubica GP. Genus Mycobacterium. In: Sneath PHA, Mair NS, Sharpe ME, Holt JG (eds), Bergey's Manual of systematic bacteriology, vol 2. Baltimore, Williams \& Wilkins Co. 1986: 1436-1457.

11. Ellner PD, Kiehn TE, Cammarata R, Hosmer M. Rapid detection and identification of pathogenic mycobacteria by combining radiometric and nucleic acid probe methods. J Clin Microbiol 1988; 26: 1349-1352.

12. Heifets L. Gen-probe tests should not be considered final in Mycobacterium tuberculosis identification. J. Clin Microbiol 1989; $27: 229$.

13. Ellner PD, Kiehn TE. Gen-probe test should not be considered final in Mycobacterium tuberculosis identification. J Clin Microbiol 1989; $27: 229$. 
